\title{
Emointelligence Algorithm Applied to SARS-COV2
}

\author{
Hafedh ABDELMELEK ${ }^{1, *}$, Amel HANINI ${ }^{1}$, Abdelhamid ADOUANI ${ }^{2}$, Amen Allah AMRI ${ }^{3}$, \\ Zihad BOUSLAMA ${ }^{4}$ \\ ${ }^{1}$ Laboratory of Integrated Physiology, Faculty of Sciences, University of Carthage, Jarzouna, Bizerte, Tunisia \\ ${ }^{2}$ Laboratory of Dynamic Systems and applications UR17ES21, University of Carthage, Jarzouna, Bizerte, \\ Tunisia \\ ${ }^{3}$ BESTConseil Office. DR Center, 13 January 1952, 7000 Bizerte, Tunisia \\ ${ }^{4}$ Laboratory of Aquatic and Terrestrial Ecology, Faculty of Sciences, University of Badji Mokhtar, BP 12, \\ 23000 Sidi Amar, Annaba, Algeria
}

*Corresponding Author: Hafedh ABDELMELEK, Laboratory of Integrated Physiology, Faculty of Sciences, University of Carthage, Jarzouna, Bizerte, Tunisia, Email: Hafedh.Abdelmelek@fsb.rnu.tn

\begin{abstract}
Our previous theories and research based on emointelligence equation suggested that Eukaryotes (Human or Animal) and Prokaryotes(bacteria or virus as SARS-COV2) could interact via different mechanisms to maintain health. Interestingly, the interpretation of emointelligence equation based on Emotion (E) and Intelligence (I) of Eukaryotes and Prokaryotes in the case of Log (EEuk/EProk) $=0$ show positive values of Log (IEuk/IProk) near 10 and EEuk/EProk=1. In addition, good health could be related to the stability of the ratio $(R)$ of E Eukaryotes/ E Prokaryotes around 1. In addition, calculation of maximum values of $(+6)$ indicate that IEuk/IProk $=10^{12}$. By contrast, when alpha have negative values $(-6)$, the equation gives IEuk/IProk=10-6. By looking for possible interaction between Eukaryotes and Prokaryotes we attempt to introduce, an innovative therapeutically tools against COVID-19 in Humans and Animals called Eukaryote-Prokaryote stability and probably explain the heterogeneity of body responses to different virus such as SARS-COV-2.
\end{abstract}

Keywords: Emotion, Intelligence; Eukaryote, Prokaryote, entanglement, SARS-COV-2.

\section{INTRODUCTION}

The present manuscript aims to propose a mathematical interpretation of emointelligence equation applied to Eukaryote and Prokaryote in the different cases of COVID-19 by i) thinking out of the box and ii) by introducing key notions at the level of neurosciences, physiology, mathematical and physical fields. Further motivations come from the failure of methodologies or theory able to understand Prokaryote-Eukaryote interactions and their implication in health and disease context. Previous research points the existence of interactions in living organisms, particularly between Animal or Human cells and viruses, bacteria (Pitkanen, 2003). Quantum biology and quantum medicine refers to a few number of Prokaryote-Eukaryote theories. Nowadays, we can consider a concept of a"quantum information: QI" 'governing the work and mechanism of living system based on emointelligence equation between Eukaryotes
(Human and Animal) and Prokaryotes (bacteria, parasites, virus like SARS-COV-2, etc) (Abdelmelek et al, 2018; Abdelmelek et al, 2019). One might envisage natural quantum mechanisms that link nature, plant, Animal, Human, and universe as previously described by our concept named One Nature One Emotion One Health where atoms and especially emotions are rearranged by entanglement mechanisms (Abdelmelek et al, 2019a, 2019b). Health could be understood as living systems coherence or harmony (Kaplan, 2001) especially the ratio Eukaryote/Prokaryote.The debate has now shifted from the question of whether quantum coherence and tunneling are involved in Prokaryote-Eukaryote mechanisms to the role played by entanglement in harmony or health.

The good health could be associated to the stability or equilibrium between Eukaryotes and Prokaryotes values giving a ratio $(\mathrm{R})$ near one. This ratio stability could be controlled by a probable entanglement between Prokaryote, 
Eukaryote, and environment. Thus, every disruption induce different ratio values such as ratio $<1$ or ratio $>1$ Interestingly, Emointelligence equation provides the basic mathematical frame work for entanglement between Prokaryote-Eukaryote and explain in part the disease genesis. The mathematical resolution of emointelligence equation could be based on three values $(\mathrm{R}<1, \mathrm{R}=1, \mathrm{R}>1)$ of the ratio of Emotion of Eukaryotes and Emotion of Prokaryotes. Emotion could modulate the interaction of Prokaryote-Eukaryote during stress, xenobiotic exposure, and electromagnetic field exposure such as WIFI, GSM or food.

Nowadays, we believe that emointelligence algorithm describe good health with aharmony between E Euk and E Prok and gives an entangled state whereas diseases results for disharmony between E Euk and E Prok and bad interactions or entanglement linked to far and near environment. When Euk cannot entangle with Prokit is impossible to cells to share the same algorithmsin order to maintain ratio near to one. The entanglement supposes that the first group Euk must be separated from the second group Prok. More generally, a pool of emotion, algorithm and health might be fundamental for interaction of Euk (Human or Animal) and Prok (SARS-COV-2) rules to maintain good health in both species. Corona virus is one of the major pathogens that primarily target the Human respiratory system. Previous outbreaks of corona viruses (CoVs) include the severe acute respiratory syndrome (SARS)-CoV and the Middle East respiratory syndrome (MERS)-CoV that have been previously characterized as agents that are a great public health threat. Interestingly, patients infected with COVID-19 showed higher body temperature of $39.0{ }^{\circ} \mathrm{C}$, leukocyte numbers, abnormal respiratory findings, and increased levels of plasma proinflammatory cytokines. Significantly high blood levels of cytokines and chemokines were noted in patients with COVID-19 infection that included IL1- $\beta$, IL1RA, IL7, IL8, IL9, IL10, basic FGF2, GCSF, GMCSF, IFN $\gamma$, IP10, MCP1, MIP1 $\alpha$, MIP1 $\beta$, PDGFB, TNF $\alpha$, and VEGFA. Some of the severe cases that were admitted to the intensive care unit showed high levels of pro-inflammatory cytokines including IL2, IL7, IL10, GCSF, IP10, MCP1, MIP1 $\alpha$, and $\mathrm{TNF} \alpha$ that are reasoned to promote disease severity [6]. In addition, SARS-COV-2 induces hypoxia associated to an increase of erythropoiesis and we note high values of red blood cells, hemoglobin, and hematocrit. Given that, leucocystosis and erythrocytosis during COVID-19 disease (Huang.et al, 2020) try to correct the total number of Euk cells compared to SARS-COV-2 number in order to maintain the ratio near 1 .

Future directions to control the spread of the disease must be based to extensive measures to reduce person-to-person transmission of SARSCOV-2 are required to control the current outbreak. Special attention and efforts to protect or reduce transmission should be applied in susceptible populations including children, health care providers, and elderly people. The early death cases of COVID-19 outbreak occurred primarily in elderly people, possibly due to a weak immune system that permits faster progression of viral infection and the ratio is low than 1 .

\section{THEORETICAL FOUNDATIONS}

Equation (1) $\mathrm{I}_{\text {Prok }}=\varepsilon \mathrm{E}_{\text {Prok }}^{\alpha} \mathrm{T}_{\text {Prok }}$ (Eq1)

Equation (2) $\mathrm{I}_{\text {Euk }}=\varepsilon \mathrm{E}_{\text {Euk }}{ }^{\alpha} \mathrm{T}_{\text {Euk }}$ (Eq2)

(Eq2)/ (Eq1)

$$
\mathrm{I}_{\text {Euk }} / \mathrm{I}_{\text {Prok }}=\mathrm{T}_{\text {Euk }} / \mathrm{T}_{\text {Prok }} *\left(\mathrm{E}_{\text {Euk }} / \mathrm{E}_{\text {Prok }}\right)^{\alpha}
$$

Constante 1 of $\mathrm{T}_{\text {Euk }} / \mathrm{T}_{\text {Prok }}=3.1510^{\wedge} 10$

$\log \quad I_{\text {Euk }} / I_{\text {Prok }}=\log \left(3.1510^{\wedge} 10\right)+\alpha \log$ $\left(\mathrm{E}_{\mathrm{Euk}} / \mathrm{E}_{\text {Prok }}\right)$

$f(x)=\log \left(3.1510^{\wedge} 10\right)+\alpha \log (x)$

I: Intelligence or Innovation, $\mathrm{T}$ : Time, E: Emotion, $\alpha$ : Factor ande: coefficient, Euk: Eukaryote, c: Prokaryote. Interestingly, the interpretation of emointelligence equation when $\log ($ EEuk/EProk $)=0$ show positive values of Log (IEuk/IProk) near 10 and EEuk/EProk=1 indicating a management of algorithm in Euk and Prok leading to good health in Human or Animal. In addition, calculation of avalues (-6, $0,+6)$ indicate that IEuk/IProk explain health or disease status(Figure 1).By looking for possible interaction between lungs, heart, intestine we attempt to introduce an innovative concept called Prokaryote and Eukaryote entanglement. Prokaryote-Eukaryote provides space-time model that correlates for algorithm stability in all emotional or intellectual processes. This present manuscript suggests that connectedness to nature or universe is one mechanism through which cells receive QI in different ways based on alpha values $(-6,0,+6)$. If alpha values are positive near +6 we have an efficient entanglement. By contrast, with negative alpha 
values near -6 we have difficult or impossible entanglement. However, recent research demonstrate the existing of signals (QI) or information that circulate only between living systems in nature ((Berman et al , 2008; Bratman et al, 2012; Abdelmelek et al, 2019) in order to explain the real significance of disease compared to health.

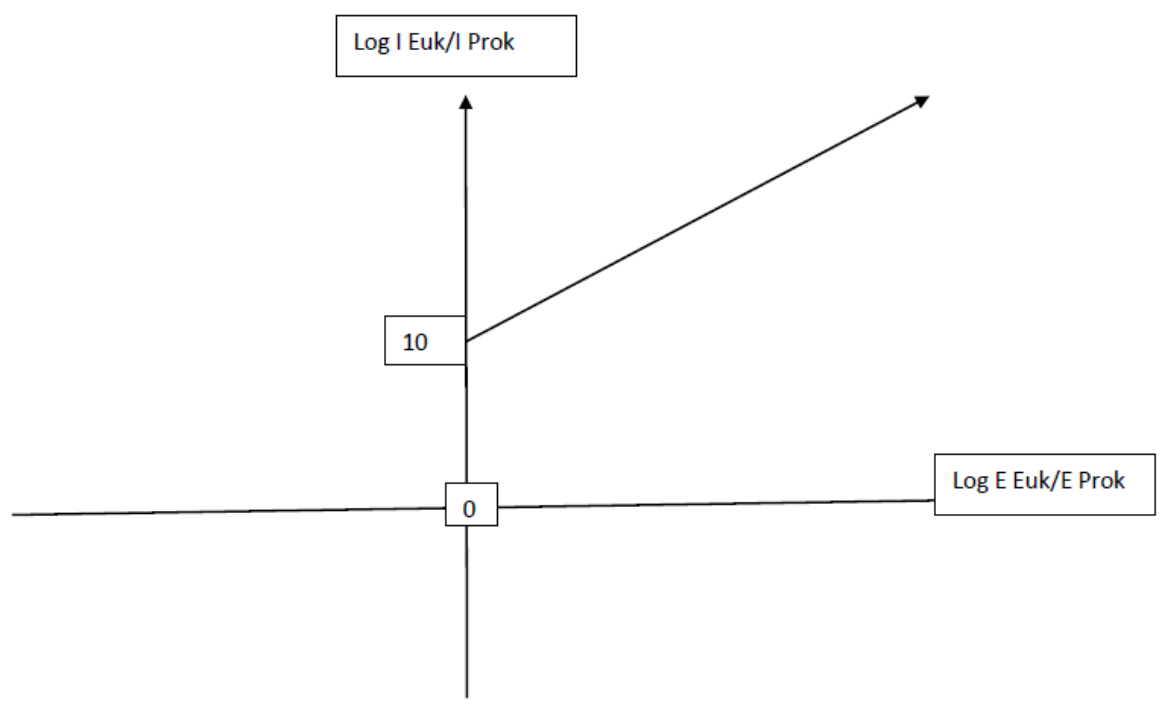

Figure1: Graphical representation of emointelligence equation applied to Prokaryote( Prok) and Eukaryote (Euk) I: Intelligence or Innovation, E: Emotion.

\section{Conclusion}

Our theories pointed to the existence of possible connectivness between Prokaryote and Eukaryoteto maintain ratio values near 1, indicating that Prokaryote-Eukaryote provides algorithm stability and health status.

\section{REFERENCES}

[1] Abdelmelek H, AmelHanini, OuajdiSouilem. Emotional Bank System and Intelligence: Emointelligence equation. J Neurobiology (2019a).

[2] AbdelmelekHafedh, AbdelhamidAdouani, Amel Hanini1, Amen Allah Amri, ZihadBouslama. Mathematical Formulation of Possible Entanglement between Human Consciousness and Unconsciousness Using Emointelligence Equation $\left(\mathrm{I}=\mathrm{T}^{*} \mathrm{E} \alpha^{*} \varepsilon\right)$ Clinical Research in Neurology Vol 2 Issue 2 (2019b).

[3] Abdelmelek H, AmelHanini, EzzedineNebil. Theories related to Emotional Bank System and
[4] Human Brain Double Door. J Neurosci Neurosurg (2018) Volume: 1.3 Manuscript ID: JNN-1-115

[5] Berman, M. G., Jonides, J., \& Kaplan, S. (2008). The cognitive benefits of interacting with nature. Psychological Science, 19(12), 1207-1212.

[6] Bratman, G. N., Hamilton, J. P., \& Daily, G. C. (2012). The impacts of nature experience on human cognitive function and mental health. Annals of the New York Academy of Sciences, 1249, 118-136.

[7] Huang C., Y. Wang, X. Li, L. Ren, J. Zhao, Y. $\mathrm{Hu}$, et al. Clinical features of patients infected with 2019 novel corona virus in Wuhan, China. Lancet, 395 (10223) (2020), pp. 497-506.

[8] Kaplan, R. (2001). The nature of the view from home: Psychological benefits. Environment and Behavior, 33(4), 507-542.

[9] Pitkanen M. TGD Inspired Theory of Prokaryote.Finland 2003; 1:6-9.

Citation: Hafedh ABDELMELEK, Amel HANINI, Abdelhamid ADOUANI, Amen Allah AMRI, Zihad BOUSLAMA. Emointelligence Algorithm Applied to SARS-COV2. ARC Journal of Neuroscience. 2020; 5(1):1012. doi: dx.doi.org/10.20431/2456-057X 0501002.

Copyright: (C) 2020 Authors. This is an open-access article distributed under the terms of the Creative Commons Attribution License, which permits unrestricted use, distribution, and reproduction in any medium, provided the original author and source are credited. 